\title{
An Upper Bound Estimate and Stability for the Global Error of Numerical Integration Using Double Exponential Transformation
}

\author{
Arezoo Khatibi ${ }^{1}$ and Omid Khatibi*2 \\ ${ }^{1}$ University of Kashan,Kashan,Iran \\ ${ }^{2}$ University of Vienna,Vienna,Austria
}

\begin{abstract}
The double exponential formula was introduced for calculating definite integrals with singular point oscillation functions and Fourier integral. The double exponential transformation is not only useful for numerical computations but it is also used in different methods of Sinc theory. In this paper we give an upper bound estimate for the error of double exponential transformation. By improving integral estimates having singular final points, in theorem 1 we prove that the method is convergent and the rate of convergence is $\mathcal{O}\left(h^{2}\right)$ where $\mathrm{h}$ is a step size. Our main tool in the proof is DE formula in Sinc theory. The advantage of our method is that the time and space complexity is drastically reduced. Furthermore, we discovered upper bound error in DE formula independent of $\mathrm{N}$ truncated number, as a matter of fact we proved stability. Numerical tests are presented to verify the theoretical predictions and confirm the convergence of the numerical solution. MSC: 65D30, 65D32.

Keyword: numerical integral, double exponential transformation, Sinc theory, quadrature formula.
\end{abstract}

\section{Introduction}

The double exponential transformations (DE) is used for evaluation of integrals of an analytic function has end point singularity. Our innovation has

*Omid Khatibi, correspond email: md_khn@yahoo.com, a1449933@unet.univie.ac.at, Faculty of Mathematics, University of Vienna, Oskar Morgenstern platz 1 1090, Vienna Austria. 
discovered the upper bound error in DE formula independent of $\mathrm{N}$ truncated number, therefore we proved stability. First we introduce double exponential transformation method. The main idea in this method change the variable by a function $\phi$ which transforms the interval of integration change to $(-\infty,+\infty)$. In section 1 we had provided background introduced the results of reference [1] and in section 3 we presents our main results in theorem 1 and provide numerical test to verify our result. Tanaka and Sugihara make full study of function classes for successful DE-Sinc approximations [2]. In [3] Ooura expanded a double exponential formula for the Fourier transform. Ooura studied animt-type quadrature formula with the same asymptotic performance as the DE-formula [4]. Several scientist studied DE-formula [5-10]. Stenger used DE Formula in Sinc approximations [9].

\section{Double Exponential Transformation}

Consider the following integral

$$
\int_{a}^{b} f(x) \mathrm{d} x
$$

where the interval $(a, b)$ is infinite or half infinite and the function under integral is analytic on $(a, b)$ and perhaps has singular point in $x=a$ or $x=b$ or both, now consider the change of variables below [11]:

$$
x=\phi(t), a=\phi(-\infty), b=\phi(+\infty),
$$

where $\phi$ is analytic on $(-\infty,+\infty)$ and

$$
I=\int_{a}^{b} f(\phi(t)) \phi \prime(t) \mathrm{d} t
$$

Such that after the change of variable the integrand decays double exponential:

$$
|f(\phi(t)) \phi \prime(t)| \approx e^{-c e^{|t|}},|t| \rightarrow \infty, c>0 .
$$

By using the trapezoidal formula with mesh size $\mathrm{h}$ on (3) we have

$$
I_{h}=h \sum_{k=-\infty}^{+\infty} f(\phi(k h)) \phi \prime(k h) .
$$


The above infinite summation is truncated from $k=-N^{-}$to $k=N^{+}$in computing (5) therefore we conclude,

$$
I_{h}^{(N)}=h \sum_{k=-N^{-}}^{N^{+}} f(\phi(k h)) \phi \prime(k h), N=N^{+}+N^{-}+1
$$

Here the $\mathrm{N}+1$ relates to zero point, for example, $N=50$ we have $N^{-}=24, N^{+}=25$ finally $50=25+24+1$. What gives us the authority to truncate $\mathrm{n}$ numbers from the infinite summation? Indeed $e^{\left(-c e^{|t|}\right)}$ quickly approaches zero. An analysis of the graph $e^{\left(-c e^{|t|}\right)}$ for small values of $\mathrm{t}$ and (4) with $\mathrm{c}=2$ is as follows Graph A shows the double exponential function.

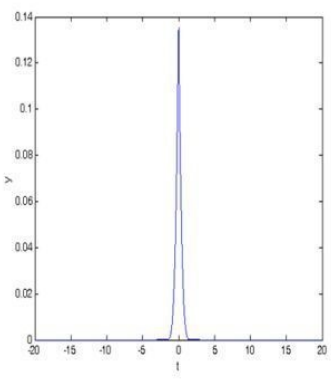

(a) Graph A

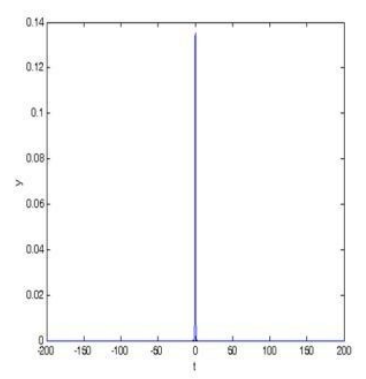

(b) Graph B

Graph B clearly shows the expansions from both side close to axis x and error growth is slow and arrives at zero as we use the double exponential formula and abbreviate it as DE. For the integral over $(-1,1)$.

$$
\int_{-1}^{1} f(x) \mathrm{d} x
$$

let

$$
\phi(t)=\tanh \left(\frac{\pi}{2} \sinh (t)\right)
$$

the transformation $x=\phi(t)=\tanh (\pi / 2 \sinh (t))$ satisfied and substitute in (5) we have

$$
\phi(t) \prime=\tanh \prime(\pi / 2 \sinh (t)) \pi / 2 \cosh (t)=\left(\frac{1}{\cosh ^{2}(\pi / 2 \sinh (t))}\right) \pi / 2 \cosh (t) .
$$

After using double exponential formula we will obtain

$$
I_{h}^{(N)}=h \sum_{k=-N^{-}}^{N^{+}} f\left(\tanh (\pi / 2 \sinh (k h)) \frac{\pi / 2 \cosh (k h)}{\cosh ^{2}\left(\frac{\pi \sinh (k h)}{2}\right)} \cdot[11]\right.
$$


In the next section, we compute the error in (5) with trapezoidal rule and mesh size h.

\section{Upper Bound Error, Convergence Rate and Sta- bility of D.E Function}

Theorem 1. $\forall h>0, h$ is step size, $c>0$ and $c$ is constant, let

$$
I=\int_{-\infty}^{\infty} f(\phi(t)) \phi \prime(t) \mathrm{d} t
$$

class function of $f$ is valid for analytic function has a singularity in first point or end point or both, $\phi$ provided by

$$
\phi(t)=\tanh \left(\frac{\pi}{2} \sinh (t)\right)
$$

such that

$$
|F(t)|=\mid f(\phi(t)) \phi(t)) \mid \approx e^{\left(-c e^{|t|}\right)},
$$

let

$$
I_{h}^{(N)}=h \sum_{k=-N^{-}}^{N^{+}} f\left(\tanh (\pi / 2 \sinh (k h)) \frac{\pi / 2 \cosh (k h)}{\cosh ^{2}\left(\frac{\pi \sinh (k h)}{2}\right)},\right.
$$

then the error estimate

$$
\left|I-I_{h}^{(N)}\right| \leq h^{2}\left(\frac{e^{-4}(1+c)}{3} e^{\frac{-c}{2}} \frac{1}{12}\left(c+c^{2}\right)\right),
$$

holds true.

Proof. The absolute error in $k^{t h}$ open interval $[\mathrm{kh},(\mathrm{k}+1) \mathrm{h}]$ using trapezoidal rule is,

$$
\begin{gathered}
E_{k}=\frac{1}{12} h^{3} M_{k}, \\
M_{k}=\sup \left|F^{\prime \prime}(X)\right|, k h \leq x \leq(k+1) h .
\end{gathered}
$$

In the next relations, the function decreases, therefore $M_{k}=F^{\prime \prime}(k h)$,

$$
\left.F(t)=f(\phi(t)) \phi^{\prime}(t)\right),
$$

thus,

$$
|F(t)| \approx e^{\left(-c e^{|t|}\right)},
$$




$$
\dot{F}(t) \approx-c e^{|t|} e^{\left(-c e^{|t|}\right)}
$$

such that,

$$
|\dot{F}(t)| \approx c e^{|t|} e^{\left(-c e^{|t|}\right)}
$$

we obtain

$$
\left|F^{\prime \prime}(t)\right| \approx c e^{|t|} e^{\left(-c e^{|t|}\right)}+c^{2} e^{2|t|} e^{\left.-c e^{|t|}\right)} .
$$

On the other hand, we know that $e^{|t|} \leq e^{2|t|}$ and by using the triangle inequality $|a+b| \leq|a|+|b|$ we have:

$$
\begin{aligned}
\left|F^{\prime \prime}(t)\right| & \leq c e^{2|t|} e^{\left(-c e^{|t|}\right)}+c^{2} e^{2|t|} e^{-c e^{|t|}}, \\
& \leq\left(c+c^{2}\right) e^{2|t|} e^{-C e^{|t|}} .
\end{aligned}
$$

We know that if $x \rightarrow \infty$ then $e^{x} \rightarrow+\infty$ and if $x \rightarrow-\infty$ then $e^{x} \rightarrow 0$; if $x \rightarrow \pm \infty$ then $|x| \rightarrow \infty$ if $c>0$ then $e^{-c|x|} \rightarrow 0$. Note that in (24), $2 t-c e^{t}$ decreases because $\frac{d}{d t}\left(2 t-c e^{t}\right)=2-c e^{t}$ and for $t>\ln \frac{2}{c}$ the derivation is negative and $k h \leq x \leq(k+1) h$, therefore, sup $\left|F^{\prime \prime}(X)\right| \leq\left(c+c^{2}\right) e^{2 k h-c e^{k h}}$, the function is also decreasing. By using (24) we have (25). We truncate the infinite summation from $-N^{-}$to $N^{+}$:

$$
\left|I-I_{h}^{(N)}\right| \leq \sum_{k=-N^{-}}^{N^{+}} E_{k} \leq \frac{1}{12} h^{3} \sum_{k=-N^{-}}^{N^{+}} M_{k} \leq \frac{1}{12} h^{3}\left(c+c^{2}\right) \sum_{k=-N^{-}}^{N^{+}} e^{2 k h-c e^{k h}} .
$$

Lemma (3.1): There exists $k_{0}$ such that if $k>k_{0}$ then,

$$
e^{2 k h-c e^{k h}}<e^{-\frac{c}{2} e^{k h}} .
$$

Proof. Multiplying $e^{\left(c e^{k h)}\right.}$ in both sides of 26 we obtain

$$
e^{2 k h}<e^{\frac{c}{2} e^{k h}} .
$$

This is enough to show that,

$$
2 k h<\frac{c}{2} e^{k h} \quad \text { or } \quad \frac{4}{c} k h<e^{k h},
$$

so, we need the following lemma with $\mathrm{t}=\mathrm{kh}$ and which means $\mathrm{a}=4 / \mathrm{c}$ can be used in next lemma . 
Lemma (3.2): For $a>0$ and for $t>2 a$ such we have $e^{t}>a t$.

Proof.

$$
e^{t}=1+\frac{t}{1 !}+\frac{t^{2}}{2 !}+\frac{t^{3}}{3 !}+\cdots>1+t+\frac{t^{2}}{2}, t>0
$$

for this we prove that

$$
\begin{gathered}
1+t+\frac{t^{2}}{2}>a t \\
1+(1-a) t+\frac{t^{2}}{2}>0,
\end{gathered}
$$

this yields

$$
t>\frac{a-1+\sqrt{(a-1)^{2}-2}}{1},
$$

also

$$
t>\frac{a-1-\sqrt{(a-1)^{2}-2}}{1},
$$

the discriminant of the quadratic equation

$$
1+(1-a) t+\frac{t^{2}}{2}=0
$$

is

$$
\Delta=(1-a)^{2}-2=a^{2}-2 a-1=0,
$$

if $\Delta \geq 0$ we put $t$. equal to the max root in quadratic equation (34),

$$
t .=a-1+\sqrt{(a-1)^{2}-2} .
$$

Then the statement is satisfied. And note that,

$$
\text { t. }<a+\sqrt{a^{2}}=2 a .
$$

If $\Delta<0$ that is the roots are complex and the coefficient of $t^{2}$ is positive then for all of $\mathrm{t}$ inequality is true. Hence we can set $t=0$ and the proof is complete. If $\Delta \geq 0$ we obtain:

$$
\begin{aligned}
& t>2 a, a=\frac{4}{c}, t=k h \Rightarrow k h>\frac{8}{c} . \\
& t<2 a, a=\frac{4}{c}, t=k h \Rightarrow k h<\frac{8}{c} .
\end{aligned}
$$


In (25) we get:

Global Error $\leq \frac{1}{12} h^{3}\left(c+c^{2}\right)\left(\sum_{k=\left[\frac{8}{c h}\right]}^{N^{+}} e^{2 k h-c e^{k h}}+\sum_{k=-N^{-}}^{\left[\frac{8}{c h}\right]} e^{2 k h-c e^{k h}}\right)$

Now we consider both sums separately.

\section{Case 1:}

$$
\frac{1}{12} h^{3}\left(c+c^{2}\right) \sum_{k h>\frac{8}{c}} e^{2 k h-c e^{k h}}
$$

Proof. Put (28) in case1 and use lemma (3.2) to set,

$$
\leq \frac{1}{12} h^{3}\left(c+c^{2}\right) \sum_{k=\left[\frac{8}{c h}\right]}^{N^{+}} e^{-\frac{c}{2} e^{k h}}
$$

On the other hand we know that for $t>0$ we have,

$$
e^{t}>1+t
$$

therefore,

$$
\begin{gathered}
e^{|k h|}>1+|k h|, \\
\sum_{\left[\frac{8}{c h}\right]}^{N^{+}} e^{-\frac{c}{2} e^{k h}} \leq \sum_{k=\left[\frac{8}{c h}\right]}^{N^{+}} e^{-\frac{c}{2}-\frac{c|k h|}{2}} \leq e^{-\frac{c}{2}} \sum_{k=\left[\frac{8}{c h}\right]}^{N^{+}} e^{-\frac{c}{2}|k h|} \leq e^{-\frac{c}{2}} \sum_{k=\left[\frac{8}{c h}\right]}^{N^{+}} e^{-\frac{c h}{2}|k|},
\end{gathered}
$$

by using the geometric series we obtain:

$$
\sum_{k=k}^{n} \frac{a^{k}-a^{n+1}}{1-a}<\frac{a^{k}}{1-a}
$$

such that

$$
\begin{gathered}
a=e^{-\frac{c h}{2}}, k .=\left[\frac{8}{c h}\right], \\
\leq e^{-\frac{c}{2}} \frac{\left(e^{-\frac{c h}{2}}\right)^{\frac{8}{c h}}}{1-e^{-\frac{c h}{2}}},
\end{gathered}
$$

moreover,

$$
\leq e^{\frac{-c}{2}} \frac{e^{-4}}{1-e^{-\frac{c h}{2}}}
$$


therefore (35) gives the following inequality:

$$
\text { Error } \leq \frac{c+c^{2}}{12} e^{\frac{-c}{2}} \frac{h^{3} e^{-4}}{1-e^{\frac{-c h}{2}}} .
$$

Lemma (3.3) : There exists $h_{0}$ such that if $h<h_{0}: e^{\frac{-c h}{2}} \leq 1-\frac{c h}{4}$.

Proof.

$$
f(h)=e^{\frac{-c h}{2}}-\left(1-\frac{c h}{4}\right),
$$

we have $f(0)=1-1=0$, and

$$
f^{\prime}(h)=\frac{-c}{2} e^{\frac{-c h}{2}}+\frac{c}{4},
$$

because $\lim _{h \rightarrow 0} e^{\frac{-c h}{2}}=1$ then

$$
\lim _{h \rightarrow 0} f^{\prime}(h)=\frac{-c}{2}+\frac{c}{4}=\frac{-c}{4},
$$

there exists $h_{0}>0$ so that if $h<h_{0}$ then $f^{\prime}(h)<0$ and $f$ decreases because $f(0)=0$ and for $h<h_{0}, f(h) \leq 0$; hence $e^{\frac{-c h}{2}} \leq 1-c h / 4$.

$$
1-e^{\frac{-c h}{2}} \geq \frac{c h}{4}
$$

we infer that

$$
\frac{1}{1-e^{\frac{-c h}{2}}} \leq \frac{1}{\frac{c h}{4}} \leq \frac{4}{c h},
$$

and

$$
\leq \frac{c+c^{2}}{12} e^{\frac{-c}{2}} \frac{h^{3}}{1-\left(1-\frac{c h}{4}\right)},
$$

thus,

$$
\leq \frac{c+c^{2}}{12} e^{\frac{-c}{2}} \frac{h^{3} \times 4}{c h}\left(e^{-4}\right)
$$

clearly

$$
\leq \frac{(1+c)}{3} e^{\frac{-c}{2}}\left(e^{-4}\right) h^{2}
$$

indeed

$$
\leq \frac{e^{-4}(1+c)}{3} e^{\frac{-c}{2}} h^{2}
$$

hence the case 1 is completed. 


\section{Case 2:}

$$
\frac{1}{12} h^{3}\left(c+c^{2}\right) \sum_{k h<\frac{8}{c}} e^{2 k h-c e^{k h}} .
$$

Proof.

$$
\begin{gathered}
2 k h-c e^{k h}<2 k h, \\
\sum_{k h<\frac{8}{c}} e^{2 k h-c e^{k h}}<\sum_{k=-N^{-}}^{\left[\frac{8}{c h}\right]} e^{2 k h}<\sum_{k=\left[\frac{8}{c h}\right]}^{-\infty}\left(e^{2 h}\right)^{k}=\sum_{k=-\left[\frac{8}{c h}\right]}^{\infty}\left(e^{-2 h}\right)^{k},
\end{gathered}
$$

we know

$$
\sum_{k=k}^{\infty} a^{k}=\frac{a^{k}}{1-a}
$$

then

$$
\sum_{k=-\left[\frac{8}{c h}\right]}^{\infty}\left(e^{-2 h}\right)^{k}=\frac{\left(e^{-2 h}\right)^{-\left[\frac{8}{c h}\right]}}{1-e^{-2 h}}
$$

so that

$$
\left[\frac{8}{c h}\right] \leq \frac{8}{c h},-\left[\frac{8}{c h}\right] \geq-\frac{8}{c h}, e^{-2 h}<1,
$$

therefore,

$$
\leq \frac{\left(e^{-2 h}\right)^{\frac{-8}{c h}}}{1-e^{-2 h}}
$$

in (40) we obtain:

$$
\left|I-I_{h}^{(N)}\right| \leq \frac{e^{-4}(1+c)}{3} e^{\frac{-c}{2}} h^{2}+\frac{1}{12} h^{3}\left(c+c^{2}\right) \frac{\left(e^{-2 h}\right)^{\frac{-8}{c h}}}{1-e^{-2 h}},
$$

in (51) consider,

$$
\frac{c h}{4}=h \Rightarrow e^{-2 h} \leq 1-h
$$

This yildes

$$
\begin{gathered}
\left|I-I_{h}^{(N)}\right| \leq \frac{e^{-4}(1+c)}{3} e^{\frac{-c}{2}} h^{2}+\frac{1}{12} h^{3}\left(c+c^{2}\right) \frac{e^{\frac{16}{c}}}{1-(1-h)}, \\
\left|I-I_{h}^{(N)}\right| \leq\left(h^{2} \frac{e^{-4}(1+c)}{3} e^{\frac{-c}{2}}+\frac{1}{12}\left(c+c^{2}\right)\right) .
\end{gathered}
$$

Now let $-N^{-}, N^{+} \rightarrow+\infty$. So we have now proved theorem 1 . 
Remark: Note that the upper bound error of theorem 1 is independent of $\mathrm{N}$ truncated number that means we proved stability. If $h \rightarrow 0$ error approaches zero and integrand method is convergence and accuracy is $\mathcal{O}\left(h^{2}\right)$.

By using this method we improve the space complexity and time complexity and we also increase the rate of convergence. In fact, we have found upper bound for global error (u.b.g.e).

\section{$4 \quad$ Numerical Result}

In the next example, utilising numerical computation and Maple software to show the result. Assume

$$
I 1=\int_{0}^{1} \exp (20(x-1)) \sin (256 x) d x .
$$

The absolute error tolerance is $10^{-8}$ where $N$ is the number of function evaluations and abs. error is the actual absolute error of the result and it gives an approximate value which is correct up to 16 significant digits.

clc

clear all

tic

$h=1 / 129 ; c=2$;

GError $=h^{2} / 3 *(1+c) *(\exp (-4-c / 2)+1 / 4 * c)$

toc

Results:

GError $=3.0451 e-005$

Elapsed time is 0.000106 seconds.

TABLE 1 comparison Comparison of the efficiency of DEFINT and ubge. The absolute error tolerance is $10^{-8}$ where $N$ is the number of function evaluations and abs. error is the actual absolute error of the result and it gives an approximate value which is correct up to 16 significant digits .

\begin{tabular}{cccc}
\hline \multicolumn{4}{c}{ DEFINT } \\
& $\mathrm{N}$ & abs. error[1] & ubge \\
\hline INTEGRAL & 259 & $4.8 \times 10^{-12}$ & $3 \times 10^{-5}$ \\
\hline$I_{1}$ &
\end{tabular}

Considering the performance of convergence rate of quadrature rules by using D.E function, the present formula is suitable for automatic integrator, 
sinc method, Fourier integral and increases the rate of convergence. It Improves the space complexity and the time complexity and has an important role in numerical analysis.

\section{References}

[1] M. Mori , M. Sugihara, The Double Exponential Transformation in Numerical Analysis, Journal Of Computational And Applied Mathematics Vol.127, (2001), 287-299.

[2] K. Tanaka, M. Sugihara, K. Murata, Function Classes for Successful DE-Sinc Approximation, Mathematics of Computation,Vol.78 , (2009), 1553-1571.

[3] T. Ooura, A Double Exponential Formula for Fourier Transform , RIMS, Kyoto Univ., Vol. 41 , (2005), 971-978.

[4] T. Ooura, Animt-Type Quadrature Formula with the Same Asymptotic Performance as the De-Formula, Journal of Computational and Applied Mathematics, Vol. 213 , (2008), 232-239.

[5] K. Horiouchi, M.Sugihara, Sinc - Galerkin Method with The Double Exponential Transformation for the Two Point Boundary Problems, Technical Report, Dept. of Mathematical Engineering, University of Tokyo , (1998), 99-105.

[6] M.Mori, A.Nurmuhammad, M.Muhammad, De-Sinc Method for Second Order Singularly Perturbed Boundary Value Problems, japan j.Indust.Appl.Math, Vol. 26 , (2009), 41-63.

[7] M. Muhammad, A. Nurmuhammad, M. Mori, Numerical Solution of Integral Equations by Means of the Sinc collocation Method Based on the Double Exponential Transformation, Journal of Computational and Applied Mathematics , Vol. 177 , (2005), 269-286.

[8] M. Sugihara, Optimality of The Double Exponential Formula Functional Analysis Approach, Numer Math, Vol. 75 , (1997).

[9] F. Stenger, Numerical Methods Based on Sinc and Analytic Functions, (1993).

[10] T. Koshihara, M. Sugihara, Optimality of The Double Exponential Formula Functional Analysis Approach, Numer Math, Vol. 75Proceedings 
Of The 1996 Annual Meeting Of The Japan Society For Industrial And Applied Mathematics , (1996).

[11] O. Khatibi, A. Khatibi, Criteria for the Application of Double Exponential Transformation, arXive, Preprints. 